\title{
Methods of the electron induced cleanning in SEM
}

Ilona Müllerová $^{1}$, Ivo Konvalina ${ }^{2}$ and Eliška Materna Mikmeková ${ }^{1}$

${ }^{1}$ Institute of Scientific Instruments of the Czech Academy of Sciences, Brno, Czech Republic, ${ }^{2}$ Institute of Scientifics Instruments of the Czech Academy of Sciences, Brno, Czech Republic

We presented, several years ago, that it is possible to obtain the images in the transmitted signal in the scanning electron microscope (SEM) at very low energies, down to several $\mathrm{eV}$ of primary electrons [1], when the deceleration mode is used in SEM [2]. One of our first results you can see in Figure 1. Commercial graphene prepared by the CVD technique (Chemical Vapor Deposition) was used for the experiments. The strong contrast of the individual graphene layers is well visible in the transmitted signal event at the energies down to $5 \mathrm{eV}$. Preliminary results were done in the ultra-high vacuum system (10-8 Pa), to avoid the contamination of the surfaces.

A special technique was developed and tested to clean the specimen and to do the imaging of the individual graphene layers, or other 2D materials, in the standard vacuum conditions of the conventional SEM. Parameters such as the primary electron beam energy, electron dose, sample bias, and scanning rate have been optimized to find the equilibrium between electron stimulated desorption (cleaning) and deposition (contamination).

When the specimen is cleaned, we can distinguish the individual graphene layers in the transmitted signal event in the SEM with standard vacuum conditions (10-4 Pa) [3]. The experimental setup and the example of trajectories of the reflected and transmitted signal electrons are shown in Figure 2a. The initial energies of electrons are $5 \mathrm{eV}$ (full lines) and $300 \mathrm{eV}$ (dashed lines) in the right half-space, and $10 \mathrm{eV}$ (full lines) and 1000 $\mathrm{eV}$ (dashed lines) in the left half-space. The initial polar angles are $0^{\circ}, 30^{\circ}, 60^{\circ}$, and $90^{\circ}$ to the optical axis. Electrostatic field strength between the detectors and the specimen is $2 \mathrm{keV} / 1 \mathrm{~mm}$.

The measurement of the transmissivity for the individual atomic layers of the graphene is shown in Figure $2 \mathrm{~b}$. The vacuum in the specimen chamber during the measurement was $5.4 \times 10-4 \mathrm{~Pa}$. The specimen was cleaned from the carbon contamination by the electron energy irradiation $700 \mathrm{eV}$ and the electron dose $0.2 \mathrm{C} / \mathrm{cm} 2$.

We can conclude that we have developed the method for the study of 2D materials, in reflected and transmitted mode in the standard SEM. We can do in-situ cleaning of the specimen e.g., from the residual PMMA (polymethyl methacrylate) or carbon contamination. The method can be used for the quantitative measurement of the individual atomic layers of 2D materials in the standard SEM. [4]. 


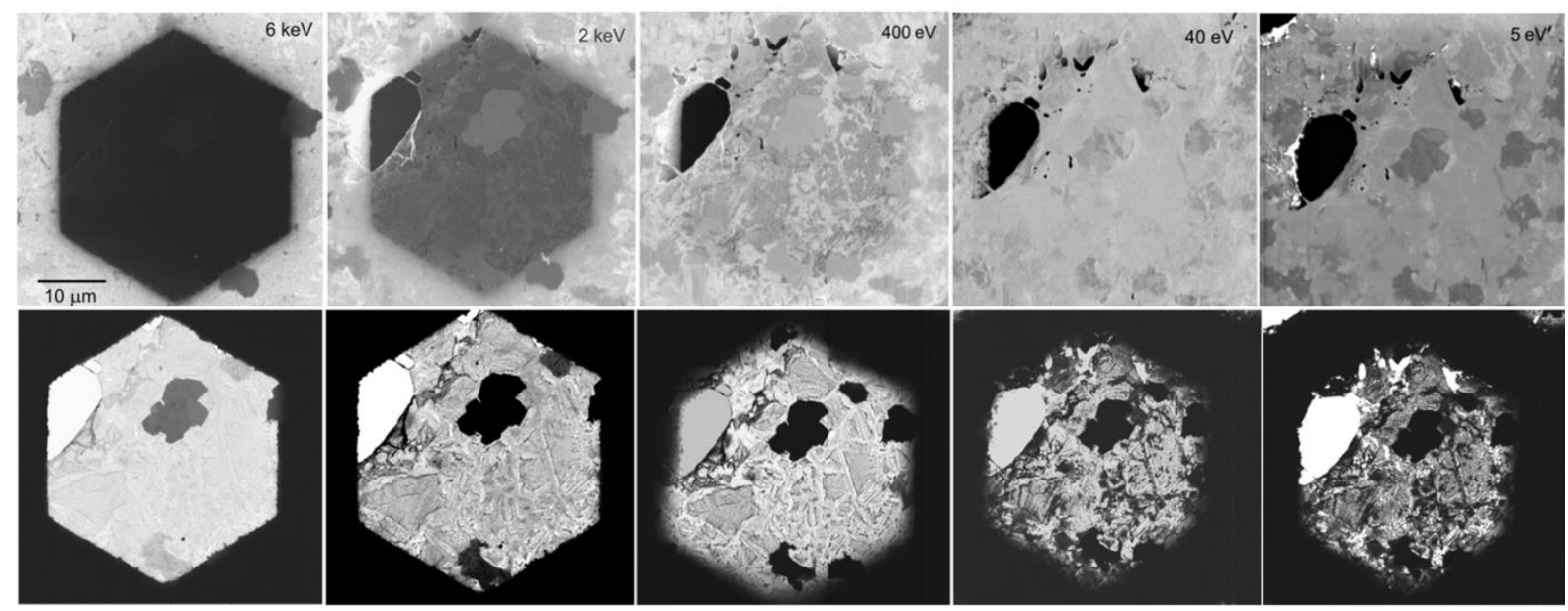

Figure 1. The imaging of the graphene in the reflected signal (upper row) and transmitted signal (bottom row). The primary beam energy from the left is $6 \mathrm{keV}, 2 \mathrm{keV}, 400 \mathrm{eV}, 40 \mathrm{eV}$, and $5 \mathrm{eV}$.

a)

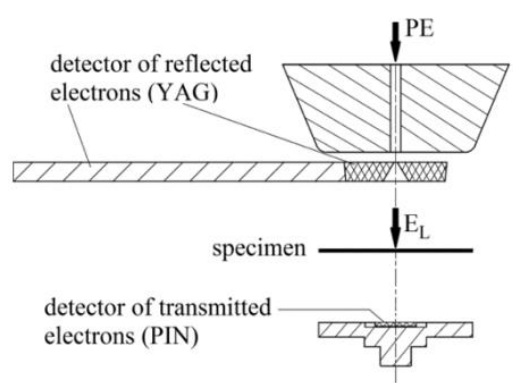

b)

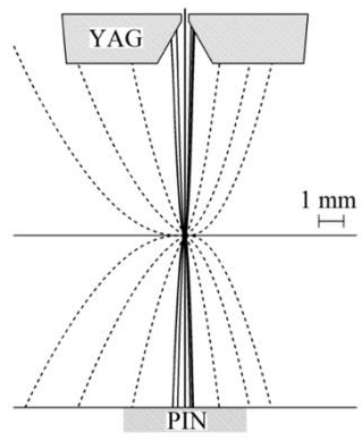

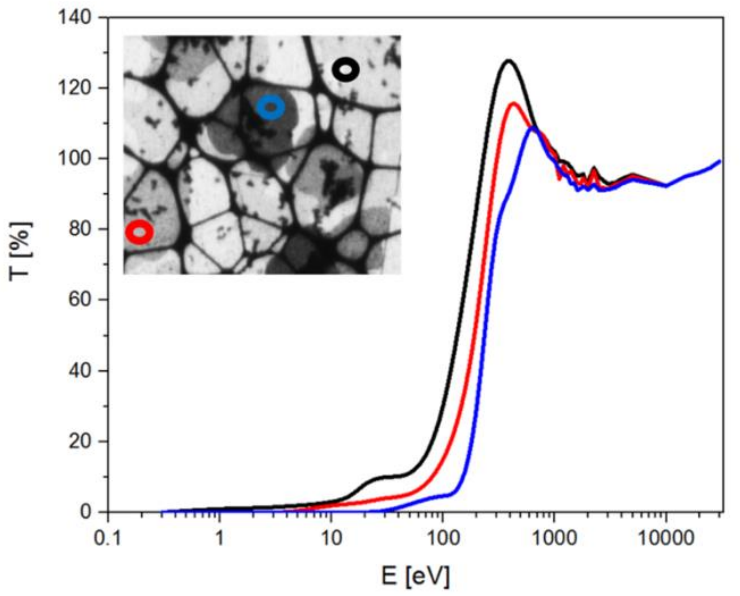

Figure 2. Basic experimental setup of the deceleration mode in SEM and simulated signal trajectories of the reflected and transmitted electrons (a). Electron energy dependence of transmissivity from one to three (from left to right curves) multilayers of the graphene measured from $30 \mathrm{keV}$ to $0.3 \mathrm{eV}$ (b).

\section{References}

[1] I Müllerová et al.: Microsc. Microanal. 21 (Suppl 3), 2015

[2] I Müllerová and L Frank, Advanced in Imaging and Electron Physics 128 (2003) p. 309

[3] E Materna Mikmeková et al.: Journal of Electron Spectroscopy and Related Phenomena 241 (2020) 146873

[4] The authors acknowledge the funding from the Technology Agency of the Czech Republic, no: TN01000008. 